\title{
Photoprocessing-driven dust evolution in the diffuse ISM
}

\author{
Lapo Fanciullo*, Vincent Guillet, Anthony Jones, François Boulanger, \\ Marc-Antoine Miville-Deschenes, Laurent Verstraete \\ IAS (Institut d'Astrophysique Spatiale) of Orsay, Paris 11 \\ E-mail: lfanciuldias.u-psud.fr, vincent.guilleteias.u-psud.fr, \\ anthony.jonesdias.u-psud.fr, francois.boulangereias.u-psud.fr, \\ marc-antoine.miville-deschenescias.u-psud.fr, \\ laurent.verstraetedias.u-psud.fr
}

\begin{abstract}
Results from the latest generation of surveys in the microwave and submillimeter domain (Planck, Herschel) suggest that the optical properties of dust in the diffuse interstellar medium are not fixed, but change from region to region, possibly due to dust evolution. Interstellar dust models need to explain this variability. The hydrogenated amorphous carbons collectively known as a$\mathrm{C}(\mathrm{H})$ are very interesting candidate dust components in this respect: their optical properties can be modified by ultraviolet photoprocessing, so that the dust properties will vary with the environmental conditions and previous dust history. We are currently working on a model containing $\mathrm{a}-\mathrm{C}(\mathrm{H})$ to determine what physical parameters can reproduce the variations of dust emission observed by Planck. We show here the effects of varying the amount of a-C(:H) photoprocessing and of carbon accretion from the gas phase.
\end{abstract}

The Life Cycle of Dust in the Universe: Observations, Theory, and Laboratory Experiments - LCDU 2013, 18-22 November 2013

Taipei, Taiwan

\footnotetext{
*Speaker.
} 


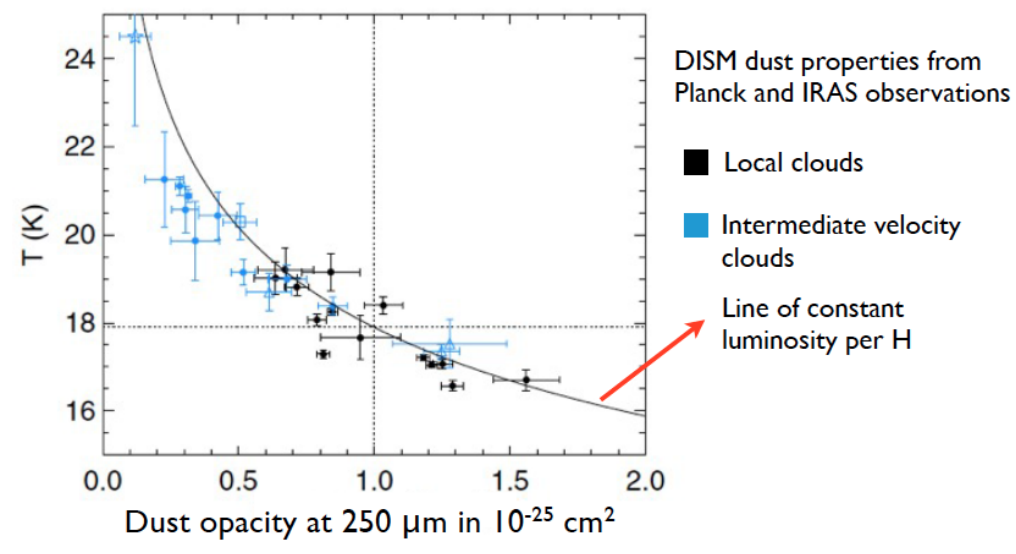

Figure 1: $T_{\mathrm{obs}}$ as a function of $\tau_{1200}$ (i.e. $\tau$ at $250 \mu \mathrm{m}$ ) for an ensemble of diffuse ISM clouds. Parameters obtained by fitting the Planck 350, 550 and $850 \mu \mathrm{m}$ bands and the IRAS $100 \mu \mathrm{m}$ band with an imposed $\beta_{\text {obs }}$ of 1.8. Image adapted from Planck Early Paper XXIV [3].

\section{Introduction}

Thermal dust emission in the sub-mm domain, which comes from grains in thermal equilibrium with the local interstellar radiation field, is empirically well-fit by a modified blackbody fit: $I_{v}=\tau_{v_{0}} B_{v}\left(T_{\mathrm{obs}}\right)\left(\frac{v}{v_{0}}\right)^{\beta_{\mathrm{obs}}}$, yielding the dust temperature $T_{\mathrm{obs}}$, the spectral index $\beta_{\mathrm{obs}}$, and the dust emissivity $\tau_{v_{0}}$ at a reference frequency $v_{0}$. Since in this work we chose $v_{0}=1200 \mathrm{GHz}$, we refer to the emissivity as $\tau_{1200}$.

It is well known that the dust emissivity in dense clouds is different from that in the diffuse interstellar medium (DISM) [1],[2], which is consistent with grain collisions forming aggregates in the dense interstellar medium (ISM). However, the Planck sub-mm survey revealed emissivity variations in the DISM [3], as can be seen in the trends followed by the observational parameters. The $T_{\mathrm{obs}}-\tau_{1200}$ anticorrelation shown in fig. 1 is close to the expected behaviour for dust emitting (i.e. absorbing) the same power per $\mathrm{H}$ atom everywhere but with varying optical properties, rather than what would be expected if the emitted spectral energy distribution (SED) followed starlight heating alone ( $\tau_{1200}$ would be similar everywhere). Environment-driven variations of optical properties are also corroborated by laboratory measurements of interstellar dust analogues [4],[5].

How can we explain intra-DISM variations? It is unlikely that aggregates could form in the DISM, where collisions are rare due to the low density, so it would be interesting to find noncollisional processes that can explain dust evolution and differentiation. In the following we explore the effects of two such processes: carbon photoprocessing and carbon accretion from the gas phase.

\section{The Jones et al. model}

A very interesting candidate dust material is hydrogenated amorphous carbon or a-C(:H), a family of materials made of aromatic ring domains (hydrogen-poor) linked by aliphatic and olefinic bridges (hydrogen-rich). Materials that are mostly aromatic are called a-C, while mostly-aliphatic 

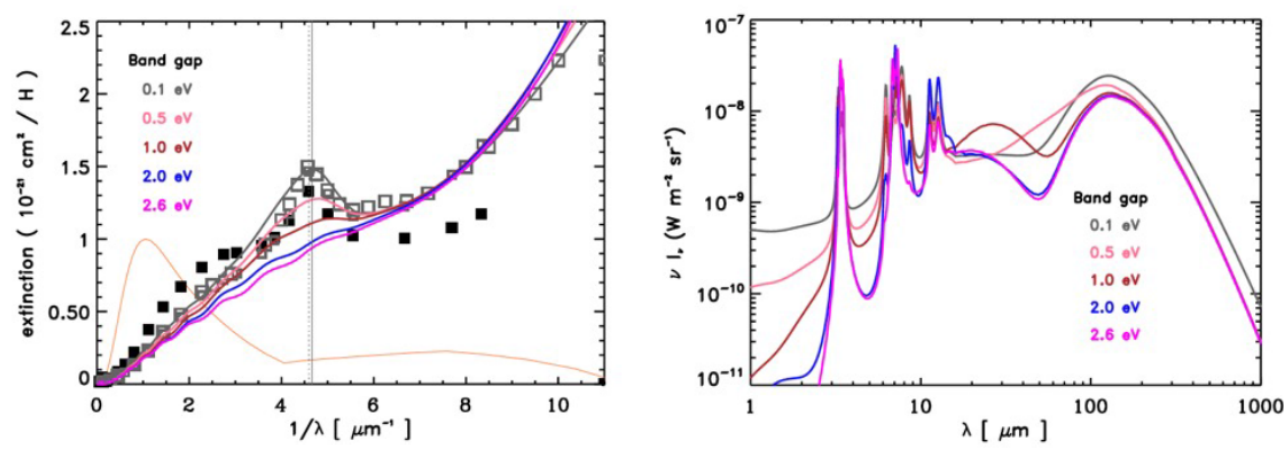

Figure 2: Variation of dust extinction curve and SED, using the Jones et al. [9] dust model, for different types of a-C(:H). In all models the band gap of the aliphatic carbon is $2.5 \mathrm{eV}$, while that of the aromatic component varies. Only the models with an a-C band gap of $\sim 0.1 \mathrm{eV}$ are physically plausible; the other ones are for illustrational purposes only. Image taken from [9].

materials are a-C:H. Recent modelling by Jones shows that the optical properties of an a-C(:H) grain can be characterized by the material's band gap, $E_{\mathrm{g}}[6],[7]$ and the grain size [8]. $E_{\mathrm{g}}$ is proportional to $\mathrm{H}$ abundance in a-C(:H); typical values are $\sim 0.1 \mathrm{eV}$ for a-C and $\sim 1 \mathrm{eV}$ for a-C(:H), with a maximum of $\sim 2.6 \mathrm{eV}$. The interesting feature of a-C(:H) is that ultraviolet (UV) photons can break its $\mathrm{C}-\mathrm{H}$ bonds, causing it to become more aromatic. a-C(:H) grains are then photoprocessed in the DISM and evolve: dust in different regions - with different processing histories - would have different properties and SEDs (Fig. 2).

To model dust evolution, we take the Jones et al. model [9] as a starting point. In this model the grains are spherical and divided into two populations: silicate (amorphous forsterite) grains with iron inclusions and a 5-nm thick a-C mantle $\left(E_{\mathrm{g}}=0.1 \mathrm{eV}\right) ; \mathrm{a}-\mathrm{C}(: \mathrm{H})$ grains, with an a-C:H core $\left(E_{\mathrm{g}}=2.5 \mathrm{eV}\right)$ and an a-C mantle $\left(E_{\mathrm{g}}=0.1 \mathrm{eV}\right)$ if their radii are $>20 \mathrm{~nm}$, and uniformly made of a-C if smaller.

In the following we explore how the predicted SED changes when we vary the $E_{\mathrm{g}}$ of a-C (to simulate varying amount of photoprocessing) and the thickness of mantles on silicates (to simulate varying degrees of accretion).

\section{Dust evolution}

With DustEM [10], we calculated the SEDs for a series of models identical to the standard Jones et al. model except that we varied the a-C band gap $(0.1-0.3 \mathrm{eV})$ and the thickness of the a-C mantles on silicates $(0-10 \mathrm{~nm})$. We then fitted the SEDs with a modified blackbody to obtain $T_{\mathrm{obs}}, \beta_{\mathrm{obs}}$ and $\tau_{1200}$ for each model. The results are shown in the top row of Fig. 3 The band gap mainly influences $\beta_{\text {obs }}$ (which is higher for less aromatic materials); while temperature is positively correlated with the amount of a-C on silicates, as to be expected since a-C is a better absorber than silicates in the UV to near-infrared range. The anti-correlation between $T_{\mathrm{obs}}$ and $\tau_{1200}$ that was evident in the observational data in Fig. 1 is not present here; overall, a combination of photoprocessing and accretion cannot explain the tendencies observed in the ISM (Sect. 1). By way of comparison, we compared these findings with changes in the dust size distribution. The 

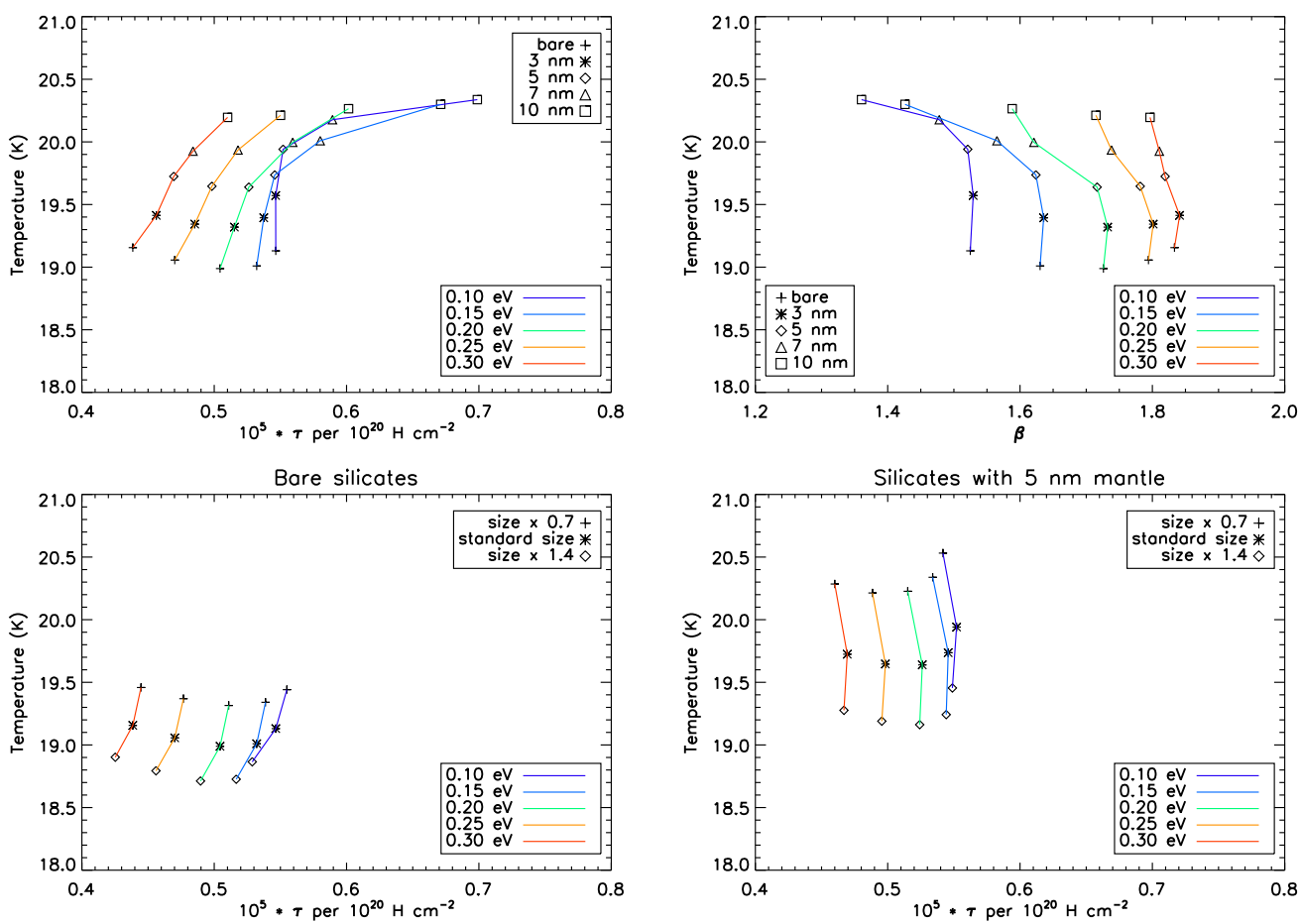

Figure 3: Simulated dust observations for the modified Jones et al. models (see text). Top row: $\tau_{1200}$ as a function of $T_{\mathrm{obs}}$ (left) and $\beta_{\mathrm{obs}}$ as a function of $T_{\mathrm{obs}}$ (right) for varying bang gap and a-C accretion. Bottom row: $\tau_{1200}$ as a function of $T_{\mathrm{obs}}$ for varying size distribution and bang gap, for a model with bare silicate grains (left) and with 5-nm a-C mantles on silicates (right). None of the attempted combinations of band gap, accretion and size distribution variability can reproduce the trends shown in Fig. 1.

bottom of Fig. 3 shows $T_{\mathrm{obs}}$ and $\tau_{1200}$ for the modified Jones et al. models where the centers of both lognormal size distributions (silicates and carbon) have been multiplied by 0.7 and 1.4. A change in size distribution only has a slight effect on temperature (increased if the silicate grains are coated in a-C) and it, too, fails to explain the tendencies described in sect. 1.

\section{Conclusions and future work}

We have shown that the tendencies followed by dust observational variables $\left(T_{\mathrm{obs}}, \beta_{\mathrm{obs}}, \tau_{1200}\right)$ in the DISM [3] seemingly cannot be explained by local variations in photo processing and gasphase accretion. It is therefore essential to explore the contribution to SED variability of other factors, such as grain shape and porosity, the effect of mixing different dust types along the line of sight and the contribution of stochastically-heated grains to the emission at the shorter wavelengths studied here.

\section{References}

[1] P. G. Martin et al., Evidence for Environmental Changes in the Submillimeter Dust Opacity, ApJ 751 28 [astro-ph/11125433] 
[2] N. Ysard et al. Modelling the dust emission from dense interstellar clouds: disentangling the effects of radiative transfer and dust properties, A\&A 542 A21 [astro-ph/12025966]

[3] Planck collaboration et al., Planck early results. XXIV. Dust in the diffuse interstellar medium and the Galactic halo, A\&A 536 A24 [astro-ph/11012036]

[4] K. Demyk et al., Variation of the FIR/submm optical properties of silicate dust analogues at low temperature: implications for the observations of interstellar cold dust emission, EAS publication series $58(405-408)$

[5] A. Coupeaud et al., Low-temperature FIR and submillimetre mass absorption coefficient of interstellar silicate dust analogues, A\&A 535 A124 [astro-ph/11092758]

[6] A. P. Jones, Variations on a theme - the evolution of hydrocarbon solids. I. Compositional and spectral modelling - the eRCN and DG models, A\&A $540 \mathrm{~A} 1$

[7] A. P. Jones, Variations on a theme - the evolution of hydrocarbon solids. II. Optical property modelling - the optEC $(s)$ model, A\&A 540 A2

[8] A. P. Jones, Variations on a theme - the evolution of hydrocarbon solids. III. Size-dependent properties - the optEC $(s)($ a) model, $A \& A \mathbf{5 4 2}$ A98

[9] A. P. Jones et al., The evolution of amorphous hydrocarbons in the ISM: dust modelling from a new vantage point, $A \& A 558$ A62

[10] M. Compiègne et al., The global dust SED: tracing the nature and evolution of dust with DustEM, A\&A 525 A103 [astro-ph/10102769] 